\title{
Sumudu Transform and Variational Iteration Method to Solve Two Point Second Order Linear Boundary Value Problems
}

\author{
Asem AL Nemrat and Zarita Zainuddin* \\ School of Mathematical Sciences, Universiti Sains Malaysia, 11800 Penang, Malaysia
}

\begin{abstract}
In this paper we propose a combined form of Sumudu Transform (ST) and the Variational Iteration Method (VIM) to solve linear two-point boundary value problems. The elegant coupling is called the Sumudu Transform Variational Iteration Method (STVIM). The results obtained are in a good agreement with the exact solution and hence this method can be regarded as a valuable tool for solving linear and nonlinear differential equations in various sciences. The proposed method has several advantages: the first one is the free choice of initial approximation with possible unknown constants, the second, is in its ability to reduce the number of computations, and finally, it avoids the round off errors and finds the solution without any linearization or discretization. Several examples confirm the reliability and efficiency of the approach, which can be used to solve other types of nonlinear boundary value problems (BVPs).
\end{abstract}

Keywords: Variational Iteration Method, Sumudu transform, General Lagrange Multiplier, Boundary Value Problems.

\section{INTRODUCTION}

In the last two decades, many analytical approximate methods have been presented to solve two-point BVPs. Most of these problems generally occur commonly in many areas of engineering, physics, chemistry, and applied mathematics. Recently, many researchers have introduced various methods to obtain approximate solutions for nonlinear differential equations (NDEs), such asVIM, which was developed by JiHuan $\mathrm{He}$ forsolving linear, nonlinear initial and BVPs (He, 1997; He, 1999; He, 2000).

It is worth mentioning that the origin of the VIM can be traced back to Inokuti, Sekine and Mura (Inokuti et. al., 1978), but the real potential of this technique was explored by $\mathrm{He}(\mathrm{He}, 1997 ; \mathrm{He}, 1999 ; \mathrm{He}, 2000)$. Moreover, He realized the physical significance of the VIM, its compatibility with the physical problems and applied this promising technique to a wide class of linear and nonlinear, ordinary, partial, differential equation, and so forth (Wazwaz, 2009; Salehpoor et al., 2010; Khan et. al.,
2012; Olayiwola et. al., 2013). This technique has been presented by many authors to be a powerful mathematical tool for solving a wide range of nonlinear operator equations (Wazwaz, 2014; Khuri \& Sayfy,2015; Ghorbani \& Bakherad, 2017; Khuri, \& Sayfy, 2017; Ahmad, 2018).The comparison of the VIM with other methods have been applied to a wide class of functional equations; (Soori, 2016; Bildik \& Deniz, 2017; Ayati \& Ahmady, 2016) and the references there in. In this modification the solution is given in an infinite series usually converging to an accurate solution (Neamaty et. al., 2015; Goswami \& Alqahtani, 2016; Singh et. al., 2017; Yun-dong\& Yi-ren, 2017; Ziane \& Cherif, 2018; Mohyud-Din et. al., 2017).

The main thrust of the proposed method is to construct a correction functional using a general Lagrange multiplier which is chosen in a proper way such that its correction solution is improved with respect to the initial trial function. None of the researchers in VIM studies has explicitly stated this fact; in numerous cases, the integral of the correction functional is a convolution, thus manipulation of Sumudu transform ought to come into place. Hence, we will introduce Sumudu correction

*Corresponding author: zarita@usm.my 
functional as an alternative and expressing the integral as a convolution. The proposed STVIM provides the solution in a rapid convergent series which may lead the solution to a closed form. In this technique, the use of Lagrange multipliers reduces the successive application of the integral operator and the cumbersome of huge computational work while still maintaining a very high level of accuracy. The proposed iterative scheme takes full advantage of VIM and preserves all the positive features of the coupled techniques. It is worth mentioning that the suggested method is applied without any linearization, discretization, and restrictive assumption and is free from round-off errors. Several examples are given to verify the accuracy and efficiency of the proposed algorithm.

The organization of this paper is as follows: the VIM, ST and the combination of ST and VIM are presented in sections 2, 3, and 4. In section 5, numerical application of the method is illustrated by three test examples to demonstrate the efficiency of the method. The conclusion is given in section 6 .

\section{VARIATIONAL ITERATION METHOD (VIM)}

To clarify the base idea of the VIM: Consider the following nonlinear differential equation:

$$
\begin{aligned}
& L(u)+N(u)-f(t)=0, \\
& B(u, \partial u / \partial t), \quad t \in \Gamma,
\end{aligned}
$$

where $\mathrm{L}$ and $\mathrm{N}$ are a linear and nonlinear operator respectively, $\mathrm{f}(\mathrm{t})$ is a known analytical function. According to the VIM, we can construct a correction functional in the following way:

$$
u_{n+1}(t)=u_{n}(t)+\int_{0}^{t} \lambda(\xi)\left(L u_{n}(\xi)+N \tilde{u}_{n}(\xi)-f(\xi)\right) d \xi,(3)
$$

where $\lambda$ is a general Lagrange multiplier, which can be identified optimally via the variational theory, the subscript $n$ denotes the $n^{\text {th }}$ approximation, and $u_{n}$ is considered as a restricted variation, i.e. $\delta \tilde{u}_{n}=0$.

\section{SUMUDU TRANSFORM (ST)}

Watugula (Watugala, 1993) introduced Sumudu transform as a new integral and is defined as:

$$
\mathrm{F}(\eta)=\mathrm{S}(\mathrm{f}(\mathrm{t}))=\int_{0}^{\infty} \frac{1}{\eta} \mathrm{e}^{-\frac{\mathrm{t}}{\eta}} \mathrm{f}(\mathrm{t}) \mathrm{dt} .
$$

In this work we used the following properties of ST:

(i) $S\left(t^{n}\right)=\mathrm{n} ! u^{n}$.

$$
\text { (ii) } S\left(f^{(n)}(t)\right)=\frac{1}{\eta^{n}} F(\eta)-\frac{1}{\eta^{n}} \sum_{k=0}^{n-1} \eta^{k} f^{(k)}(0) \text {. }
$$

where $f^{(0)}(0)=f(0), f^{(k)}(0), k=1,2,3, \ldots, n-1$ are the $k^{\text {th }}$ derivative of $f(t)$, and $S(f(t))=F(\eta)$. If $F(\eta)$ is the Sumudu transform of $f(t)$, then $f(t)$ is called the inverse Sumudu transform of $F(\eta)$ and is expressed by $f(t)=S^{-1}\{F(\eta)\}$, where the inverse Sumudu transform operator is $S^{-1}$.

\section{SUMUDU TRANSFORMVARIATIONAL ITERATION METHOD(STVIM)}

In a wide range of problems that appear in the literature, the general form of Lagrange multiplier is found to be of the form:

$$
\lambda=\lambda(t-\xi) .
$$

In this article, we will make the assumption that $\lambda$ is expressed in this way. In such a case, the integration is basically the convolution; hence ST is appropriate to use. Applying ST on both sides of (3) the correction functional will be constructed in the following manner:

$$
\begin{aligned}
& S\left(u_{n+1}(t)\right)=S\left(u_{n}(t)\right)+ \\
& \quad S\left(\int_{0}^{t} \lambda(\xi)\left(L u_{n}(\xi)+N \tilde{u}_{n}(\xi)-f(\xi)\right) d \xi\right),
\end{aligned}
$$

therefore

$$
\begin{aligned}
& S\left(u_{n+1}(t)\right)=S\left(u_{n}(t)\right)+ \\
& S(\lambda(t)) * S\left(L u_{n}(t)+N \tilde{u}_{n}(t)-f(t)\right), \\
& \quad=S\left(u_{n}(t)\right)+ \\
& \eta S(\lambda(t)) S\left(L u_{n}(t)+N \tilde{u}_{n}(t)-f(t)\right) .
\end{aligned}
$$

To find the optimal value of $\lambda=\lambda(t-\xi)$, we first take the variation with respect to $u_{n}(t)$. Thus:

$$
\begin{aligned}
& \frac{\delta}{\delta u_{n}} S\left(u_{n+1}(t)\right)=\frac{\delta}{\delta u_{n}} S\left(u_{n}(t)\right)+ \\
& \eta \frac{\delta}{\delta u_{n}} S(\lambda(t)) S\left(L u_{n}(t)+N \tilde{u}_{n}(t)-f(t)\right) .
\end{aligned}
$$

and hence upon applying the variation this simplifies to 


$$
S\left(\delta u_{n+1}(t)\right)=S\left(\delta u_{n}(t)\right)+\eta S(\lambda(t)) S\left(\delta u_{n}(t)\right)
$$

We assume that $\mathrm{L}$ is a linear differential operator with constant coefficients given by

$$
\begin{aligned}
L(u) \equiv & a_{n} u^{(n)}+a_{n-1} u^{(n-1)}+a_{n-2} u^{(n-2)}+\ldots \\
& +a_{2} u^{\prime \prime}+a_{1} u^{\prime}+a_{0} u,
\end{aligned}
$$

where $a_{n}{ }^{\prime} s$ are constants. It is important to note that if the coefficients contain only non-constant terms of the form $t^{k}$, then the Sumudu variational approach is still valid. The ST of the first term of the operator $\mathrm{L}$ is given by

$$
S\left(a_{n} u^{(n)}\right)=\frac{a_{n}}{\eta^{n}} S(u)-\frac{a_{n}}{\eta^{n}} \sum_{k=0}^{n-1} \eta^{k} u^{(k)}(0),(12)
$$

so the variation with respect to $u$ is

$$
\delta S\left(a_{n} u^{(n)}\right)=\frac{a_{n}}{\eta^{n}} S(\delta u) .(13)
$$

The other terms in the operator L, namely

$a_{n-1} u^{(n-1)}+\ldots+a_{1} u^{\prime}, a_{0} u$, yield similar results. Hence using (13), Eq. (10) reduces to

$$
\begin{aligned}
S\left(\delta u_{n+1}(t)\right)= & S\left(\delta u_{n}(t)\right)+ \\
& \eta S(\lambda(t))\left(\sum_{k=0}^{n-1} \frac{a_{n}}{\eta^{n}}\right) S\left(\delta u_{n}(t)\right), \\
= & \left(1+\eta S(\lambda(t))\left(\sum_{k=0}^{n-1} \frac{a_{n}}{\eta^{n}}\right)\right) S\left(\delta u_{n}(t)\right) .
\end{aligned}
$$

The extremum condition of $u_{n+1}$ requires that

$\delta u_{n+1}=0$. This means that the right-hand side of Eq. (14) should be set to zero. Hence, we have the stationary condition

$$
S(\lambda(t))=-\frac{1}{\sum_{k=0}^{n} \frac{a_{n}}{\eta^{n-1}}} .
$$

Taking the Sumudu inverse of the last equation gives the optimal value of $\lambda$. For this value of $\lambda$, we have the following iteration formulation:

$$
\begin{aligned}
& S\left(u_{n+1}(t)\right)=S\left(u_{n}(t)\right)+ \\
& \quad S\left(\int_{0}^{t} \lambda(t-\xi)\left(L u_{n}(\xi)+N \tilde{u}_{n}(\xi)-f(\xi)\right) d \xi\right),
\end{aligned}
$$

\section{CASE STUDIES}

Example I: Consider the following linear differential equation (Opanuga et al., 2017): with the boundary conditions

$$
u(0)=0, \quad u(1)=e-1 .
$$

The exact solution of the problem is $u(1)=e-1$.By applying the STVIM to find a solution for Eq.(17), we construct the variational iteration correction functional as the following:

$$
\left.u_{n+1}(t)=u_{n}(t)+\int_{0}^{t} \lambda(t-\xi)\left(u_{n}^{\prime \prime}(\xi)-u_{n}(\xi)-1\right) d \xi\right) .
$$

Next, by applying ST, we have:

$$
\begin{aligned}
S\left(u_{n+1}(t)\right)= & S\left(u_{n}(t)\right)+ \\
& \left.S\left(\int_{0}^{t} \lambda(t-\xi)\left(u_{n}^{\prime \prime}(\xi)-u_{n}(\xi)-1\right) d \xi\right)\right) .
\end{aligned}
$$

or equivalently, by applying the convolution property, we get:

$$
\begin{aligned}
S\left(u_{n+1}(t)\right)= & S\left(u_{n}\right)+S(\lambda) * S\left(u_{n}^{\prime \prime}-u_{n}-1\right), \\
= & S\left(u_{n}\right)+\eta S(\lambda) S\left(u_{n}^{\prime \prime}-u_{n}-1\right), \\
= & S\left(u_{n}\right)+\eta S(\lambda)\left(\left(\frac{1}{\eta^{2}}-1\right) S\left(u_{n}\right)\right. \\
& \left.-\frac{u_{n}(0)}{\eta^{2}}-\frac{u_{n}^{\prime}(0)}{\eta}-1\right) .
\end{aligned}
$$

Applying the variation on the Eq. (21) with respect to $u_{n}(t)$ , we get:

$$
\begin{aligned}
\frac{\delta}{\delta u_{n}} S\left(u_{n+1}(t)\right)= & \frac{\delta}{\delta u_{n}} S\left(u_{n}\right)+\frac{\delta}{\delta u_{n}} \eta S(\lambda) \times \\
& \left(\left(\frac{1}{\eta^{2}}-1\right) S\left(u_{n}\right)-\frac{u_{n}(0)}{\eta^{2}}-\frac{u_{n}^{\prime}(0)}{\eta}-1\right) .
\end{aligned}
$$

By simplifying Eq. (22), we get:

$$
\begin{aligned}
S\left(\delta u_{n+1}(t)\right) & =S\left(\delta u_{n}\right)+\eta S(\lambda)\left(\frac{1}{\eta^{2}}-1\right) S\left(\delta u_{n}\right), \\
& =S\left(\delta u_{n}\right)\left(1+\eta S(\lambda)\left(\frac{1-\eta^{2}}{\eta^{2}}\right)\right) .
\end{aligned}
$$

The extremum condition of $u_{n+1}(t)$ requires that $\delta u_{n+1}(t)=0$, then

$$
\begin{gathered}
S\left(\delta u_{n}\right)\left(1+S(\lambda)\left(\frac{1-\eta^{2}}{\eta}\right)\right)=0, \\
S(\lambda)=\frac{\eta}{\eta^{2}-1} .
\end{gathered}
$$

Applying the inverse of ST, we get:

$$
\lambda(t)=-\sinh (t) .
$$

Substituting Eq. (25) into Eq. (20), we get: 


$$
\begin{aligned}
S\left(u_{n+1}(t)\right)= & S\left(u_{n}(t)\right)- \\
& \left.S\left(\int_{0}^{t} \sinh (t-\xi)\left(u_{n}^{\prime \prime}(\xi)-u_{n}(\xi)-1\right) d \xi\right)\right) \\
= & S\left(u_{n}(t)\right)- \\
& \eta S(\sinh (t)) S\left(u_{n}^{\prime \prime}(t)-u_{n}(t)-1\right)
\end{aligned}
$$

Suppose that $u_{0}(t)=u(0)+u^{\prime}(0) t$, since $u(0)=0$ and $u^{\prime}(0)=A$, then:

$$
\begin{aligned}
S\left(u_{1}(t)\right) & =S\left(u_{0}(t)\right)-\eta S(\sinh (t)) S\left(u_{0}^{\prime \prime}(t)-u_{0}(t)-1\right), \\
& =S(A t)-\eta S(\sinh (t)) S(-A t-1) .
\end{aligned}
$$

Applying the inverse of ST, we have

$$
u_{1}(t)=A \sinh (t)+\cosh (t)-1 .
$$

By using the boundary condition $u(1)=e-1$, then $A=1$. So $u_{1}(1)=e^{t}-1$., which is the exact solution for our problem.

Example II: Consider the following linear differential equation (Khuri, \& Sayfy, 2012):

$$
u^{\prime \prime}+u+t=0, \quad 0 \leq t \leq 1,
$$

with the boundary conditions

$$
u(0)=0, \quad u(1)=0 .
$$

The exact solution of the problem is $u(1)=\csc (1) \sin (t)-t$. By applying the STVIM to find a solution for Eq. (29), we construct the variational iteration correction functional as the following:

$$
\left.u_{n+1}(t)=u_{n}(t)+\int_{0}^{t} \lambda(t-\xi)\left(u_{n}^{\prime \prime}(\xi)+u_{n}(\xi)+t\right) d \xi\right) .(31)
$$

Next, by applying ST, we have:

$$
\begin{aligned}
S\left(u_{n+1}(t)\right)= & S\left(u_{n}(t)\right)+ \\
& \left.S\left(\int_{0}^{t} \lambda(t-\xi)\left(u_{n}^{\prime \prime}(\xi)+u_{n}(\xi)+\xi\right) d \xi\right)\right) .
\end{aligned}
$$

or equivalently, by applying the convolution property, we get:

$$
\begin{aligned}
S\left(u_{n+1}(t)\right)= & S\left(u_{n}\right)+S(\lambda) * S\left(u_{n}^{\prime \prime}+u_{n}+t\right), \\
= & S\left(u_{n}\right)+\eta S(\lambda) S\left(u_{n}^{\prime \prime}+u_{n}+t\right), \\
= & S\left(u_{n}\right)+\eta S(\lambda)\left(\left(\frac{1}{\eta^{2}}+1\right) S\left(u_{n}\right)\right. \\
& \left.-\frac{u_{n}(0)}{\eta^{2}}-\frac{u_{n}^{\prime}(0)}{\eta}+\eta\right) .
\end{aligned}
$$

Applying the variation on the Eq. (33) with respect to $u_{n}(t)$, we get:

$$
\begin{aligned}
\frac{\delta}{\delta u_{n}} S\left(u_{n+1}(t)\right)= & \frac{\delta}{\delta u_{n}} S\left(u_{n}\right)+\frac{\delta}{\delta u_{n}} \eta S(\lambda) \times \\
& \left(\left(\frac{1}{\eta^{2}}+1\right) S\left(u_{n}\right)-\frac{u_{n}(0)}{\eta^{2}}-\frac{u_{n}^{\prime}(0)}{\eta}+\eta\right) .
\end{aligned}
$$

By simplifying Eq. (34), we get:

$$
\begin{aligned}
S\left(\delta u_{n+1}(t)\right) & =S\left(\delta u_{n}\right)+\eta S(\lambda)\left(1+\frac{1}{\eta^{2}}\right) S\left(\delta u_{n}\right) \\
& =S\left(\delta u_{n}\right)\left(1+\eta S(\lambda)\left(1+\frac{1}{\eta^{2}}\right)\right)
\end{aligned}
$$

The extremum condition of $u_{n+1}(t)$ requires that $\delta u_{n+1}(t)=0$, then

$$
\begin{gathered}
S\left(\delta u_{n}\right)\left(1+S(\lambda)\left(\frac{1+\eta^{2}}{\eta}\right)\right)=0, \\
S(\lambda)=-\frac{\eta}{\eta^{2}+1} .
\end{gathered}
$$

Applying the inverse of ST, we get:

$$
\lambda(t)=-\sin (t)
$$

Substituting Eq. (37) into Eq. (32), we get:

$$
\begin{aligned}
S\left(u_{n+1}(t)\right) & =S\left(u_{n}(t)\right)- \\
& \left.S\left(\int_{0}^{t} \sin (t-\xi)\left(u_{n}^{\prime \prime}(\xi)+u_{n}(\xi)-\xi\right) d \xi\right)\right), \\
& =S\left(u_{n}(t)\right)-\eta S(\sin (t)) S\left(u_{n}^{\prime \prime}+u_{n}+t\right) .
\end{aligned}
$$

Suppose that $u_{0}(t)=u(0)+u^{\prime}(0) t$, since $\mathrm{u}(0)=0$ and $u^{\prime}(0)=A$, then

$$
\begin{aligned}
S\left(u_{1}(t)\right) & =S\left(u_{0}(t)\right)-\eta S(\sin (t)) S\left(u_{0}^{\prime \prime}(t)+u_{0}(t)+1\right), \\
& =S(A t)-\eta S(\sin (t)) S(A t+t) .
\end{aligned}
$$

Applying the inverse of ST, we have

$$
u_{1}(t)=(A+1) \sin (t)-t .
$$

By using the boundary condition $u(1)=0$, then $A=\csc (1)-1$.So $u_{1}(1)=\csc (1) \sin (t)-t$, which is the exact solution for our problem.

Example III: Consider the following linear differential equation (Chun \& Sakthivel, 2010):

$$
u "-u=\cos (t), \quad 0 \leq t \leq 1,
$$

with the boundary conditions 


$$
u(0)=0, \quad u(1)=0 .
$$

The theoretical solution is

$$
\begin{gathered}
u(1)=\frac{-3 \cosh (1)+3 \sinh (1)+\cos (1)+2}{4 \sinh (1)} e^{t}+ \\
\frac{3 \cosh (1)+3 \sinh (1)-\cos (1)-2}{4 \sinh (1)} e^{-t}-\frac{\cos (t)}{2} .
\end{gathered}
$$

By applying the STVIM to find a solution for Eq. (41), we construct the variational iteration correction functional as the following:

$$
\left.u_{n+1}(t)=u_{n}(t)+\int_{0}^{t} \lambda(t-\xi)\left(u_{n}^{\prime \prime}(\xi)-u_{n}(\xi)-\cos (\xi)\right) d \xi\right) .(43)
$$

Next, by applying ST, we have:

$$
\begin{aligned}
S\left(u_{n+1}(t)\right)= & S\left(u_{n}(t)\right)+ \\
& \left.S\left(\int_{0}^{t} \lambda(t-\xi)\left(u_{n}^{\prime \prime}(\xi)+u_{n}(\xi)+\xi\right) d \xi\right)\right) .
\end{aligned}
$$

or equivalently, by applying the convolution property, we get:

$$
\begin{aligned}
S\left(u_{n+1}(t)\right) & =S\left(u_{n}\right)+S(\lambda) * S\left(u_{n}^{\prime \prime}-u_{n}-\cos (t)\right), \\
= & S\left(u_{n}\right)+\eta S(\lambda) S\left(u_{n}^{\prime \prime}-u_{n}-\cos (t)\right), \\
= & S\left(u_{n}\right)+\eta S(\lambda)\left(\left(\frac{1}{\eta^{2}}-1\right) S\left(u_{n}\right)\right. \\
& \left.-\frac{u_{n}(0)}{\eta^{2}}-\frac{u_{n}^{\prime}(0)}{\eta}-S(\cos (t))\right) .
\end{aligned}
$$

Applying the variation on the Eq. (45) with respect to $u_{n}(t)$, we get:

$$
\begin{aligned}
\frac{\delta}{\delta u_{n}} S\left(u_{n+1}(t)\right)= & \frac{\delta}{\delta u_{n}} S\left(u_{n}\right)+\frac{\delta}{\delta u_{n}} \eta S(\lambda) \times \\
& \left(\left(\frac{1}{\eta^{2}}-1\right) S\left(u_{n}\right)-\frac{u_{n}(0)}{\eta^{2}}-\frac{u_{n}^{\prime}(0)}{\eta}-S(\cos (t))\right) .
\end{aligned}
$$

By simplifying Eq. (46), we get:

$$
\begin{aligned}
S\left(\delta u_{n+1}(t)\right) & =S\left(\delta u_{n}\right)+\eta S(\lambda)\left(\frac{1}{\eta^{2}}-1\right) S\left(\delta u_{n}\right), \\
& =S\left(\delta u_{n}\right)\left(1+\eta S(\lambda)\left(\frac{1}{\eta^{2}}-1\right)\right) .
\end{aligned}
$$

The extremum condition of $u_{n+1}(t)$ requires that $\delta u_{n+1}(t)=0$, then

$$
\begin{gathered}
S\left(\delta u_{n}\right)\left(1+S(\lambda)\left(\frac{\eta^{2}-1}{\eta}\right)\right)=0, \\
S(\lambda)=-\frac{\eta}{\eta^{2}-1} .
\end{gathered}
$$

Applying the inverse of ST, we get:

$$
\lambda(t)=-\sinh (t) .
$$

Substituting Eq. (49) into Eq. (44), we get:

$$
\begin{aligned}
S\left(u_{n+1}(t)\right)= & S\left(u_{n}(t)\right)- \\
& \left.S\left(\int_{0}^{t} \sinh (t-\xi)\left(u_{n}^{\prime \prime}(\xi)-u_{n}(\xi)-\cos (\xi)\right) d \xi\right)\right) \\
= & S\left(u_{n}(t)\right)- \\
& \eta S(\sinh (t)) S\left(u_{n}^{\prime \prime}(t)-u_{n}(t)-\cos (t)\right) .
\end{aligned}
$$

Suppose that $u_{0}(t)=u(0)+u^{\prime}(0) t$, since $u(0)=1$ and $u^{\prime}(0)=A$, then

$$
\begin{array}{r}
S\left(u_{1}(t)\right)=S\left(u_{0}(t)\right)-\eta S(\sinh (t)) S\left(u_{0}^{\prime \prime}(t)-u_{0}(t)-\cos (t)\right) \\
=S(1+A t)-\eta S(\sinh (t)) S(-1-A t-\cos (t)) \\
=1+A \eta+\frac{\eta^{2}}{\eta^{2}-1}\left(-1-A \eta-\frac{1}{\eta^{2}+1}\right)
\end{array}
$$

Applying the inverse of ST, we have

$$
u_{1}(t)=\frac{3 \cosh (t)}{2}-\frac{\cos (t)}{2}+A \sinh (t)
$$

By using the boundary condition $u(1)=1$, then:

$$
A=\frac{e^{-i}\left(-3 e^{i}+e+4 e^{1+i}+e^{1+2 i}-3 e^{2+i}\right)}{2\left(-1+e^{2}\right)} .
$$

Substituting the value of A into (52), the obtained results were found to be very close to the theoretical solution with some truncation error:

$$
\begin{aligned}
u_{1}(1)= & \frac{-3 \cosh (1)+3 \sinh (1)+\cos (1)+2}{4 \sinh (1)} e^{t}+ \\
& \frac{3 \cosh (1)+3 \sinh (1)-\cos (1)-2}{4 \sinh (1)} e^{-t}-\frac{\cos (t)}{2} .
\end{aligned}
$$

\section{CONCLUSIONS}

In this paper, STVIM has been efficiently applied for solving two-point boundary value problems to give rapid convergent successive approximations without any linearization, discretization or restrictive assumptions that may change the physical behaviour of the problem and absorb the positive features of the coupled techniques. Hence, one important feature of the proposed approach is that the Sumudu correction functional is introduced that enables the integral to be expressed, in many instances in the form of a convolution. ST will make the variational problems easier to tackle, particularly in finding the general Lagrange multiplier. Furthermore, the STVIM method 
facilitates the computational work and makes the solution converges rapidly to the exact solution. For nonlinear problems where the exact solution does not exist, only a few numbers of approximations are required for numerical purpose. In all the cases considered, we have obtained excellent performances that may lead to a promising approach in solving the nonlinear two-point BVPs and could find wide applications.

\section{ACKNOWLEDGEMENT}

The authors gratefully acknowledge the financial assistance provided by Universiti Sains Malaysia under the Research University grant scheme 1001/PMATHS/8011041.

\section{REFERENCES}

Ahmad, H., 2018, Variational iteration method with an auxiliary parameter for solving differential equations of the fifth order. Nonlinear Sci. Lett. A, 9(1), pp.27-35.

Ayati, Z. and Ahmady, S., 2016, The comparison of optimal homotopy asymptotic method and homotopy perturbation method to solve Fisher equation. Computational Methods for Differential Equations, 4(1), pp.43-53.

Bildik, N. and Deniz, S., 2017, January. Modification of perturbation-iteration method to solve different types of nonlinear differential equations. In AIP Conference Proceedings (Vol. 1798, No. 1, p. 020027). AIP Publishing.

Chun, C. and Sakthivel, R., 2010, Homotopy perturbation technique for solving two-point boundary value problems comparison with other methods. Computer Physics Communications,181(6), pp.1021-1024.

Ghorbani, A. and Bakherad, M., 2017, A variational iteration method for solving nonlinear Lane Emden problems. New Astronomy, 54, pp.1-6.

Goswami, P. and Alqahtani, R.T., 2016, On the solution of local fractional differential equations using local fractional Laplace variational iteration method. Mathematical Problems in Engineering, 2016.

He, J.H., 1997, Semi-inverse method of establishing generalized variational principles for fluid mechanics with emphasis on turbomachinery aerodynamics.
International Journal of Turbo and Jet Engines, 14(1), pp.23-28.

He, J.H., 1999, Variational iteration method a kind of nonlinear analytical technique: some examples. International journal of non-linear mechanics, 34(4), pp.699-708.

He, J.H., 2000, Variational iteration method for autonomous ordinary differential systems. Applied Mathematics and Computation, 114(2-3), pp.115-123.

Inokuti, M., Sekine, H. and Mura, T., 1978, General use of the Lagrange multiplier in nonlinear mathematical physics. Variational method51ASM Science Journal Special Issue 2018(1) in the mechanics of solids, 33(5), pp.156-162.

Khan, Y., Vazquez-Leal, H., Hern, L. and Faraz, N., 2012, Variational iteration algorithm-II for solving linear and non-linear ODEs. International Journal of Physical Sciences, 7(25), pp.3099-4002.

Khuri, S.A. and Sayfy, A., 2012, A Laplace variational iteration strategy for the solution of differential equations. Applied Mathematics Letters,25(12), pp.22982305 .

Khuri, S.A. and Sayfy, A., 2015, A novel fixed-point scheme: Proper setting of variational iteration method for BVPs. Applied Mathematics Letters, 48, pp.75-84.

Khuri, S.A. and Sayfy, A., 2017, Generalizing the variational iteration method for BVPs: Proper setting of the correction functional. Applied Mathematics Letters, 68, pp.68-75. 
Mohyud-Din, S.T., Sikander, W., Khan, U. and Ahmed, N., 2017, Optimal variational iteration method using Adomians polynomials for physical problems on finite and semi-infinite intervals. The European Physical Journal Plus, 132(5),p.236.

Neamaty, A., Agheli, B. and Darzi, R., 2015, Variational iteration method and Hes polynomials for time fractional partial differential equations. Progress in Fractional Differentiation and Applications, 1(1), pp.47-55.

Olayiwola, M.O., Gbolagade, A.W. and Akinpelu, F.O., 2013, Variational iteration method for the solution of boundary value problems. World Acad. Sci. Eng. Technol, 7, pp.122.

Opanuga, A.A., Owoloko, E.A., Okagbue, H.I. and Agboola, O.O., 2017, Finite difference method and Laplace transform for boundary value problems.

Salehpoor, E., Jafari, H. and Afrapoli, M.A., 2010, Revised variational iteration method for solving systems of ordinary differential equations. Applications and Applied Mathematics (ISSN: 1932-9466), 1, pp.110-121.

Singh, R., Das, N. and Kumar, J., 2017, The optimal modified variational iteration method for the Lane-Emden equations with Neumann and Robin boundary conditions. The European Physical Journal Plus, 132(6), p.251.

Soori, M., 2016, A Comparison between the Variational Iteration Method and the Homotopy Perturbation Method for the Burgers-Huxley Equation.

Watugala, G.K., 1993, Sumudu transform: a new integral transform to solve differential equations and control engineering problems. Integrated Education, 24(1), pp.35-43.

Wazwaz, A.M., 2009, The variational iteration method for analytic treatment for linear and nonlinear ODEs. Applied Mathematics and Computation, 212(1), pp.120134.

Wazwaz, A.M., 2014, The variational iteration method for solving linear and nonlinear ODEs and scientific models with variable coefficients. Central European Journal of Engineering, 4(1), pp.64-71.

Yun-dong, L. and Yi-ren, Y., 2017, Vibration analysis of conveying fluid pipe via Hes variational iteration method. Applied Mathematical Modelling, 43, pp.409-420.

Ziane, D. and Cherif, M.H., 2018, Variational iteration transform method for fractional differential equations. Journal of Interdisciplinary Mathematics, 21(1), pp. 185199. 